\title{
Students with Learning Disabilities at University
}

\section{Sandra Zecchi}

Università degli Studi di Firenze, Italy.

\begin{abstract}
Specific Learning Disorders involve a plurality of functions that impact the decoding of the alphabetic code. They have an evolutionary character, that is, vary according to the age of the subject.

The present work, connected to positive practical experiences, investigates the characteristics of DSAs in adulthood and the impact with university teaching. It presents the outcomes in progress of an interdisciplinary project (including pedagogical, medical and engineering area).

Learning Disabilities ( $L D)$ disorder are relatively new for the education at the university level, and have therefore pushed teachers and researcher to define research projects, both scientific and pedagogic, aimed to suit the needs of $L D$ students. In such perspective, due to the high variability of LD manifestations and degrees and also to the peculiar students' features, a multidisciplinary approach and strategies are required to identify personalized educational paths for LD students while respecting, the specificity and the objectives of the different university courses.
\end{abstract}

Keywords: Learning Disabilities; Inclusive Education; Special Education needs; Didactic tools; University. 


\section{Premise}

This paper refers to an experimental procedure carried out by a research team at the Florence University (ITA).

LD problems are relatively new for the education at the university level, and have therefore pushed teachers and researcher to define research projects, both scientific and pedagogic, aimed to suit the needs of LD students. In such perspective, due to the high variability of LD manifestations and degrees and also to the peculiar students' features, a multidisciplinary approach and strategies are required to identify personalized educational paths for LD students while respecting, the peculiarity and the objectives of the different university courses.

\section{Project plan}

The Italian national education system, due to the Law 170/2010 "Nuove norme in materia di Disturbi Specifici di Apprendimento in ambito scolastico", have to apply inclusive protocols for student with disabilities. These protocols have to be adopted by schools and universities and encourage the use of teaching methodologies and strategies for students with special needs in order to promote their school success and to ensure the educational processes.

The increasing number of university students with LD is a consequence of the education tutelage promoted by the law 170/2010. As a consequence, there is a need of specific support strategies and personalized teaching models that allows to use compensative tools and assessment modalities, suitable for specific study courses.

The research project "Students with Learning Disabilities at University - Realization of a Protocol for Usability of Teaching and Individual Study" is part of this kind of approach.

The project began in January 2015. Its purpose is to ensure a full application of education law for university students with LD. Its specific objective is to create a teaching-management protocol for the inclusion of students with LD inside the Florence University.

It has been possible to reach this objective thanks to an interdisciplinary approach which offers an integrate view of the object of research, on the perspective of the opportunities and the criticalities. This is the reason why the research team members have different competencies, with pedagogical, sanitary and engineering skills. This allowed, from the early steps of the project, the integration of different observation levels in order to understand and fit the needs of students with LD from several points of view and to develop aimed to the achievement of their welfare within the University path. 


\subsection{Project phases}

During the first step a study of the previous data of students with LD enrolled at the University of Florence and a detailed research on the state of the art have been performed. This step also enabled the research team to create a group of students to be involved in the project in order to better acquire, in a structured manner, their needs and requests.

This project has involved different partners, including various local associations.

At the end of this step, a group of 43 students provided their contacts in order to participate to the project research initiatives.

During the second step a data collection and a student specific needs analysis were performed, thanks to the presentation meeting, an on-line questionnaire for the students and four focus group took place. The results of these steps are presented in the following paragraph.

At the end of the final steps of the Project, the following outcomes will be delivered:

- $\quad$ a University Guide Lines for teachers, university staff and students. It should offer good praxis and indications useful for student's career, educational offer, teaching activities and university services efficiency;

- a web site, which will be part of the institutional University site, built according to the usability and universal design standards, suitable for students with LD. The web site will also be addressed to teachers, university staff and students and should offer information and suggestions about services, requests, assistive materials given by the University to students with LD. In particular, it should help the students from the beginning to the end of their university career and the teachers to have an effective approach with students with LD.

The project is currently in progress. The initial operating phase is concluded, and revision processes are in progress.

\section{Tools}

\subsection{Questionnaire}

From the earliest meetings of the working group it arises the idea to submit a questionnaire to students with Learning Disabilities. Starting from the research plan, this kind of entry survey would have permitted us to reach rapidly a wide number of goals:

- $\quad$ to establish a first interaction with students;

- $\quad$ to have an early feedback from students about the actual aims of the project, based on the number and type of the received responses; 
- to collect information regarding the personal student's experiences, in order to better organize the following steps of the project;

- $\quad$ to test the students' Learning Disabilities knowledge and awareness, in order to have a future comparison on the same topics at the end of the project;

- $\quad$ to use the questionnaire as a test itself in order to verify, where possible, the layout and contents compliance level.

The survey was titled "Inquiry on students with Learning Disabilities in the University of Florence” and the questions had been grouped in the following sections:

- “Biographical Information”, in which information about age, gender, year and kind of university course and other previous educational experiences were requested;

- "Diagnosis and treatment", where the students were asked to indicate the kind of Learning Disabilities, the presence of co-morbidities and the age of the first Learning Disabilities diagnosis;

- "Degree of satisfaction of various aspects of university life", where the students were asked to evaluate different aspects of their university life as burocracy, lessons, entrance test and exams organization, usability of the university web sites, quality of learning material;

- "Relationships inside the University", where the students were asked to evaluate their degree of satisfaction in their relationship with professors, university personnel and other students;

- "Supports available in University", where the students were asked to evaluate the supports provided from the University: the CESPD Learning Disabilities help desk and the tutoring services;

- "Tools and instruments", where the students were asked to evaluate their use frequency of technological tools and software able to support their Learning Disabilities and the degree;

- "Individual strategies", the students were asked to evaluate the efficacy of their personal strategies used to improve the learning;

- "Feedback", the students were asked to give a feedback about the questionnaire itself, starting from their specific issues, giving an assessment of the relevance of the content, the chosen language and the layout.

A total of 24 questions were submitted to participants: mainly multiple-choice ones and, only where necessary, open-ended questions. The aim was to achieve easily grouped and 
quantifiable answers, together with examples, motivations and student's personal observations, otherwise undetectable.

For the multiple-choice questions, a four-value Likert scale was used, where the choice of a response with an increasing value always corresponded to more positive item evaluations.

The questionnaire was anonymous and it was submitted online to a chosen group of students with different Learning Disabilities, using an email invitation containing the link to the questionnaire web page, a personal password and some synthetic compilation instructions. Further explanations and clarifications were also provided to students during the first meeting with the research group in which the whole project was presented.

The questions were formulated using, whenever possible, a Learning Disabilities friendly language: short sentences; coordinated sentences rather than subordinate ones; grouped questions for thematic areas; simple vocabulary.

For the same reason, the questionnaire layout was created according to the following rules: large, sans serif fonts; targeted use of capital and bold letters to emphasize the logical structure of the text; alignment of the text to the left; no use of hyphenation; choice of not too contrasting colors for text and background, (no black characters on a white background).

\subsection{Questionnaire results}

The invitation to participate to the questionnaire was sent to a group of 43 students enrolled at the University of Florence with a learning disability certification. We obtained 26 answers (60\% of the total amount).

Analyzing the results, the main critical items are the following:

- $\quad$ the University of Florence web site;

- $\quad$ the online and paper forms;

- the exams and test worksheets.

The degree of satisfaction about the relationship with professors, university personnel and other students is high.

The questionnaire received a positive feedback from the students.

\subsection{Focus groups}

Once analyzed the results of the questionnaire, the investigations on the needs of students with Learning Disabilities continued by means of four focus groups. The aim was to investigate some of the most critical items perceived as a priority by the students, which, at 
the same time, are found to be actually improvable elements during the following steps of the Project. As a result, the following areas of analysis were identified:

- the website of the University: when the students with Learning Disabilities were asked about their degree of satisfaction on various aspects of their university life, it received the lowest mean. The decision to carry out a further study on the website is also linked to the fact that one of the final outcomes of the project will be a DSAfriendly website;

- the relationship with the professors: although judged satisfactory in terms of interpersonal relationships, and in terms of teaching support tools had the higher number of issues in the open-ended responses, the relation with teachers revealed a widespread behavior heterogeneity;

- the teaching support tools: the most relevant results were, surprisingly, not the level of satisfactory use but rather the low level of knowledge and use by the students themselves, in many cases less than $50 \%$ of the total students responding to the questionnaire;

- the support services provided by the University: students with Learning Disabilities, often did not know their existence or, in many cases, judged them unsatisfactory.

For collecting data during the focus groups it was decided to use a method typical of the Quality Function Deployment (QFD), a tool originally used for quality management, whose versatility makes it easy to use in a number of different contexts.

Specifically, the used method was a simplified correlation matrix, a grid where, after identifying the customer (in this case the focus group participating students, with the guidance of some moderators) and the critical elements (the so-called VOC, voice of costumer) relating to the scope of analysis, the possible solutions (so-called CTQ, critical to quality) for each VOC are defined. The result is a matrix in which each VOC is associated with one or more CTQ. During the four focus groups, moderators and students have therefore worked in teams to build four correlation matrices, one for each of the chosen areas. The matrix definition was made in real time, using a wide screen to display all the steps.

The time for each focus group was limited to 30 minutes, including introduction, VOC collection and reorganization (to eliminate repetitions, not relevant items, etc.), CTQ collection and final construction of the matrices. Obviously all matrices were further ordered in a more organized way in a subsequent debriefing, exclusively reserved to moderators.

Each focus group was attended by two moderators, two observers and a working group of 8 students. 
The analysis of the correlation matrices permitted to better define the following project steps towards some specific items, directly obtained from the CTQ analysis. In particular, the main results could be summarized as follows:

- $\quad$ the legislation alone is not enough to ensure that students with Learning Disabilities a full integration within their university career. The laws must be associated to guidelines and to good practices handbook;

- $\quad$ there is a need to invest in training and information processes, able to "educate" all users to relate properly with each other and with the available educational tools;

- as a consequence, a homogeneous behavior policy has to be promoted in the relationship between teachers and students: teachers and students need to know what they can get one from each other but also what are the limits of their possible requests;

- a website that collects guidelines and good practices handbook is mandatory, it must be structured in several levels of interpretation (for teachers, for students, for the university personnel involved with students) and above all it must be easily usable by the "weaker" users, the students with Learning Disabilities themselves, with ad hoc contents and layout;

- $\quad$ some compensatory easy-to-use instruments must be provided, not affected by a technological obsolescence, which effectively would make them quickly unusable.

\section{Conclusions}

From the survey questionnaire and focus groups we can already deduce the important considerations for further work, although it is only the half the project.Five years after Law 170/2010 University institutions are not yet fully able to match the educational needs of LD students' study. But even these students aren't sometimes aware of their rights. We need invest in global educational processes at all institutional level in order to spread knowledge about the needs of people with LD in adulthood.

The University has a specific responsibility in this direction, it must have teachers trained, and they have to know the rules and the results of studies with scientific evidence around the theme of LD and their characteristics in adulthood. The teachers have to be able to implement a university teaching LD-friendly and know how to adopt specific protocols to which all students with LD can be accessed directly in the form systematized and not just occasionally and sporadically. This is in fact the purpose of this project. 


\section{References}

Dehaene S. (2009). I neuroni della lettura. Milano: Raffaello Cortina.

Dettori F. (2015). Né asino, ne prigro, sono dislessico. Esperienze scolastiche e universitarie di persone con DSA. Milano: Franco Angeli.

Dolan R. P., Hall T.E. (2001). Universal design for learning: implications for large-scale assessment. IDA Perspectives, 27 (4), 22-25.

Fink R. P. (1998). Literacy development in successful men and women with dyslexia. Annals of Dyslexia, 48, 311-346.

Genovese E. (Eds.). (2010). Dislessia e Università. Trento: Erickson.

Genovese E., Ghidoni E., et al. (2011). Dislessia nei giovani adulti. Trento: Erickson.

Gérard C.L. (2011). Cliniques des troubles des apprentissages. De l'évaluation neuropsychologique à la programmation éducative. Bruxelles: De Boeck.

Hatcher J. et al. (2002). Cognitive assessment of dyslexic students in higher educatio. British Journal of educational Psychology, 72, 119-133.

Lami L. et al. (2008), Evoluzione del profilo di lettura della dislessia. Studio longitudinale su un gruppo di dislessici divenuti giovani adulti. Dislessia, 5 (1), 7-17.

Marzocchi G. M. et al. (2011). La presa in carico dei bambini con ADHD e DSA. Trento: Erickson.

Olofsson A. et al. (2012). Learning and study strategies in university students with dyslexia: implications for teaching. Procedia Social and behavorial Sciences, 47, 1184-1193.

Pannetier E. (2010). Comprendre la dyslexie. Genève: Ambre Editions.

Reid A. A. et al. (2006). Cognitive Profiles of adult developmental Dyslexics: Theoretical implications. Wiley InterScience, 13, 1-24.

Trisciuzzi L., Zappaterra T. (2014). La dislessia. Una didattica speciale per le difficoltà di lettura e scrittura. Milano: Guerini e Associati. 